\title{
A model of human behavior based on visual information processing mechanism for reliability-based system design
}

\author{
Kimihiro Yamanaka \\ Dept. of System Design of Tokyo Metropolitan University, Tokyo, Japan
}

Email address:

kiyamana@sd.tmu.ac.jp (K. Yamanaka)

To cite this article:

Kimihiro Yamanaka. A Model of Human Behavior Based on Visual Information Processing Mechanism for Reliability-Based System Design, Psychology and Behavioral Sciences. Vol. 2, No. 2, 2013, pp. 20-27. doi: 10.11648/j.pbs.20130202.11

\begin{abstract}
The aim of this study is to discuss the relation between the reliability of structural systems and the performance of human perception in human-machine systems. In this paper, we would like to evaluate the effect of performance deterioration on structural safety from the viewpoint of uncertainties in human behavior. The sensitivity of structural safety to such uncertainties is discussed on the basis of a stress-strength model by considering that uncertainties in structural systems can be divided into objective and subjective uncertainties in applied stress and strength. There are two types of subjective uncertainties, one associated with the designer and the other associated with the operator. In this context, the performance of the operator in terms of visual perception was investigated through visual tasks. Concretely, two kinds of experiments such as response time and useful field of view were carried out. Based on experimental results and analysis, it was shown that the relations between subjective uncertainty of machine and human performance affect the final probability of failure of structural systems, including human-machine systems.
\end{abstract}

Keywords: Reliability, Structural Safety, Human Performance, Response Time, Useful Field Of View

\section{Introduction}

The important factor of subjective uncertainty in the evaluation on structural safety is based on human error. The analysis of human error provides useful information required for the promotion of efficient management of structural systems, including human-machine systems, since structural failures are mostly due to poor human performance, for example: lack of perception, omissions, and mistakes of recognition or judgment. To address these problems, a number of human reliability analysis models have been proposed thus far by specialists from various fields such as psychology, natural science, behavioral or social science, and cognitive science [1-3]. However, the demand for such models can be understood if a distinction is made between various instances of the models.

There are three types of responses in human behavior under the operator model [1], where the operator receives perceptive stimulation. One type is a skill-based response, which usually refers to an unconscious reaction. In this case, the operator can react within the shortest possible response time after receiving some information, in which case reactive patterns are created without the process of recognition, identification, and judgment. The second type of response is known as rule-based response, which is based on information processes such as recognition of the situation and matching the task to the situation. The third type of response is known as knowledge-based response, whose main features are the processes of identification and judgment. Since the latter two types of reactions are conscious reactions, the response time is usually longer than in the case of unconscious reactions due to the intermittent processes of identification, judgment, and prediction.

In this paper, we discuss the relation between human performance and structural safety by considering objective and subjective uncertainties. Therefore, the effect of human performance on the reliability of human-machine systems is considered from the viewpoint of cognitive science and psychophysiology in ergonomics. In addition, we focus on the parameters of visual perception, such as the width of the visual range, the response time (RT), and the useful field of view (UFOV), within which useful information for recognition is received, as well as the response to visual stimuli. It is important to understand that the performance deterioration of human visual information processing can degrade the reliability of human-machine systems. 


\section{Reliability of Human-Machine Sys- tems}

\subsection{Structural Safety and Human Performance}

The relation between structural safety and human behavior in human-machine systems is shown in Fig. 1 in the form of a block diagram. In human-machine systems, the reliability (or structural safety) is strongly dependent on human performance. While the most pervasive model of human performance has been the stimulus-organism-response (S-O-R) paradigm it is no longer widely accepted as valid [2]. A metaphor is a way of conveying a specific meaning, that is, to gain understanding of human cognition. One metaphor that has become widespread recently is the view of humans as an information processing system (IPS) [3]. The traditional view of information processing has been extended to the step ladder model (SLM). In IPS models, the internal mechanisms are typically discussed in far greater detail than in S-O-R models. In the same context, focusing on the organism part of the paradigm, the step ladder model includes the function of three types of IPS models, such as skill-based, rule-based, and knowledge-based IPS, as developed by Rasmussen [1].

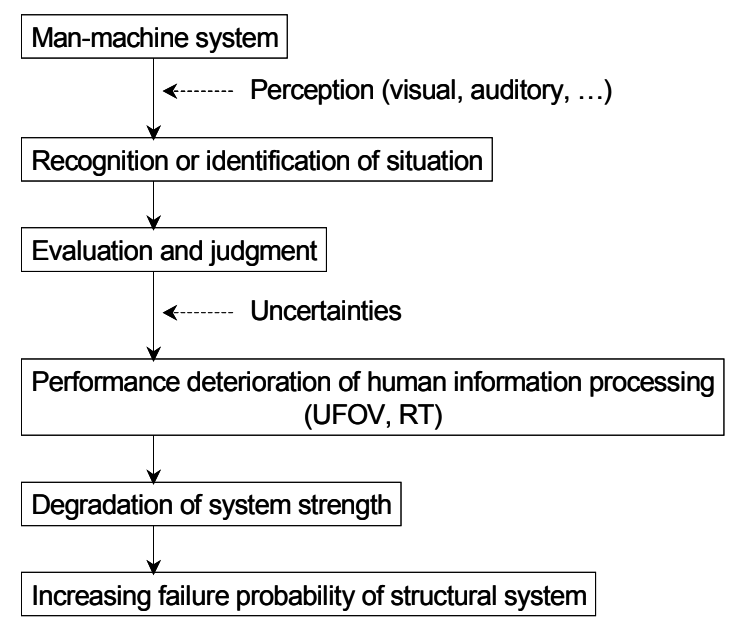

Figure 1. Structural safety and human performance.

The response to external information obtained through sensory perception is typically illustrated by the operator model proposed by Rasmussen. When receiving external information pertaining to safety in the form of visual or auditory signals, the response to these signals is described by the human performance model in Fig. 2. As shown in the figure, there are various patterns of response to external information.

Human performance is an important factor in human-machine systems, when information is perceived with objective uncertainty with respect to the perception stimuli, because the response and safety depend on the judgment by the human operator and the accuracy of recognizing external information. Therefore, a decrease in performance with respect to perceptual information processing, such as decreased UFOV or increased response time, might affect the system strength in human-machine systems, resulting in an increase in the probability of failure of structural systems, including human-machine systems.

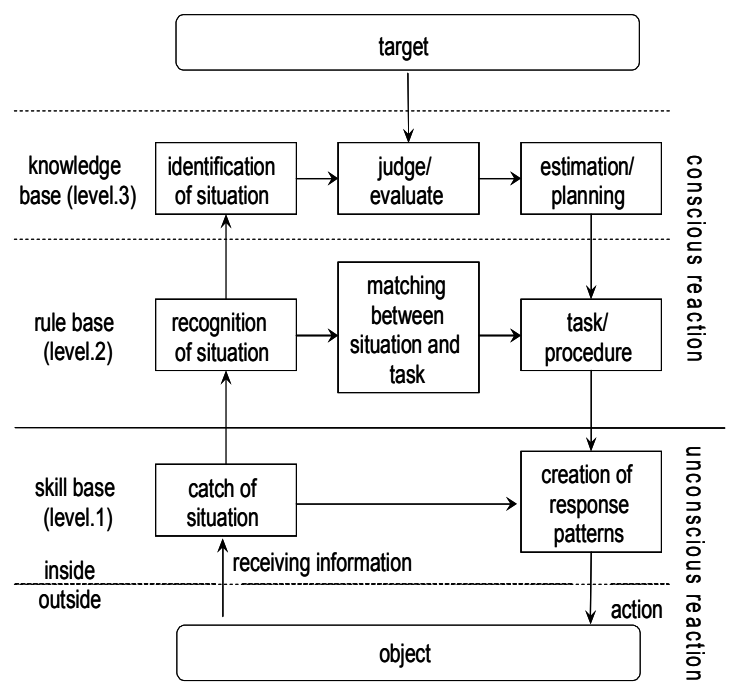

Figure 2. Operator model [1].

\subsection{Effect of Performance Deterioration on Structural Safety in Stress-Strength Model}

To use the stress-strength model to formulate the concept of structural safety, including in the case of human-machine systems, stress applied to structural systems can be illustrated using the strength of the structural system [5], as shown in Fig. 3. It is well known that the probability of failure is defined by

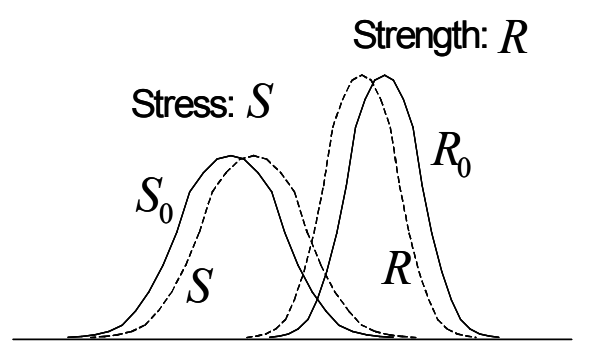

Figure 3. Stress strength model [5].

$$
P_{f}=P[R<S]
$$

where $R$ and $S$ are the applied stress and strength and $P[x]$ denotes the occurrence probability of event $x$. An important point here is that the applied stress and strength must be formulated as random variables with objective and subjective uncertainties. When $R_{0}$ and $S_{0}$ denote the original random variables with objective uncertainties, as shown in Fig. 3 , the critical state is expressed as

$$
R_{0}<N S_{0}
$$

In Eq. (2), $N$ denotes the subjective uncertainties where

$$
N=N_{S} \bullet N_{R}
$$


Therefore, the probability of failure $P_{f}$ can be rewritten as

$$
P_{f}=P\left[R_{0}<N S_{0}\right]
$$

If the structural system is a human-machine system, $R_{0}$ is expressed as

$$
R_{0}=R_{m}+R_{h}
$$

where $R_{m}$ and $R_{h}$ denote machine strength and human performance, respectively, which correspond to the proof strength or strength capacity with respect to the applied stress. From Eqs. (3) and (4), the probability of failure of a structural system characterized by the parameter of human strength capacity can be estimated from

$$
P_{F}=P\left[R_{0} / N_{R} \leq N_{S} S_{0}\right]=P\left[R_{m} / N_{R}+R_{h} / N_{R} \leq N_{S} S_{0}\right]
$$

The factor $N_{R}$ in Eq. (6) denotes the subjective uncertainty of structural strength of the human-machine system. Although it is difficult to evaluate $N_{R}$, it incorporates the subjective uncertainties of the designer and the operator, namely

$$
N_{R}=f\left(N_{D}, N_{o}\right)
$$

$N_{D}$ and $N_{R}$ denote the respective subjective uncertainties of the designer and the operator, where the former indicates human error in the form of omissions and misunderstanding of the design, while the latter stems from the lack of external information input, such as visual perception. However, it should be noted that it is difficult to determine the function in Eq. (7) since these uncertainties are associated with each processing step in Fig. 4.

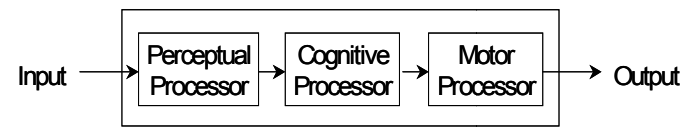

Figure 4. Uncertainties lay behind each process.

\section{Case Study on Human Performance in Term of RT \& UFOV}

This section introduces experimental results obtained by the authors as a case study on visual perception and human behavior in order to discuss the aspects of subjective uncertainty. The experiment examined two aspects of human performance namely, response time (RT) and UFOV, which is the visual region of cognition.

\subsection{Response Time [14,15]}

The schematics of the experimental devices are shown in Fig. 5. The response time was measured as the time necessary for recognizing a visual stimulus in an electromagnetically shielded room. A 21" CRT display (GDM-F500, SONY Corporation) was installed at a distance of $600 \mathrm{~mm}$ from the eyes of the participant, and the NTSC signal output from an imposer was converted into an RGB signal by an up-scan converter.

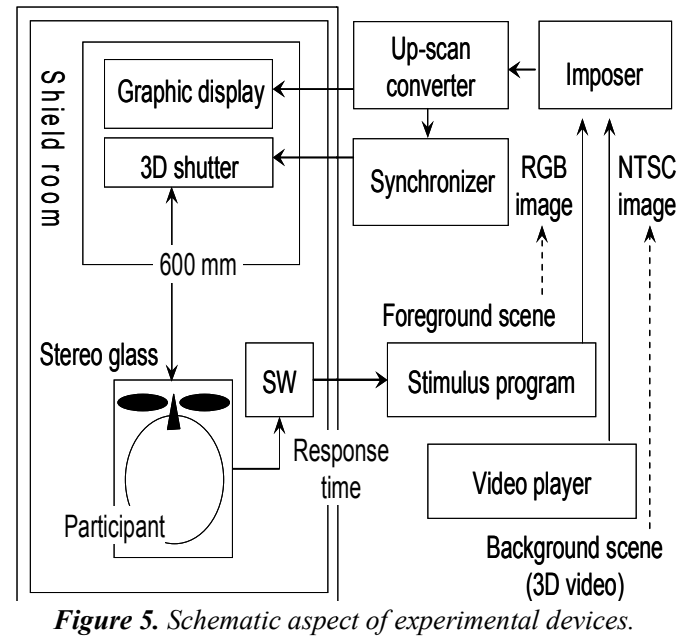

In the experiment for response time, the visual stimulus consists of foreground and background scenes, as shown in Fig. 6. In the foreground scene, S1 (a simple white circle with a size of $0.36^{\circ}$ flashing at 53 cycles $/ \mathrm{min}$ ) was used to keep the visual attention in the center. The target mark S2, also with a size of $0.36^{\circ}$, was a specific shape from the Snellen chart, and a similar shape was used as a standard mark. The appearance ratio of the target mark to the standard mark was 0.25 , and the interval between S1 and S2 was 1700 $\mathrm{ms}$, where $\mathrm{S} 2$ appeared within a zone with a radius between $0^{\circ}$ and $11.70^{\circ}$ and random coordinates. Furthermore, the participants were provided with two buttons, as shown in Fig. 6 . The subjects were instructed to press the button on the right as soon as they recognized the target mark S2 and the button on the left when S1 disappeared.

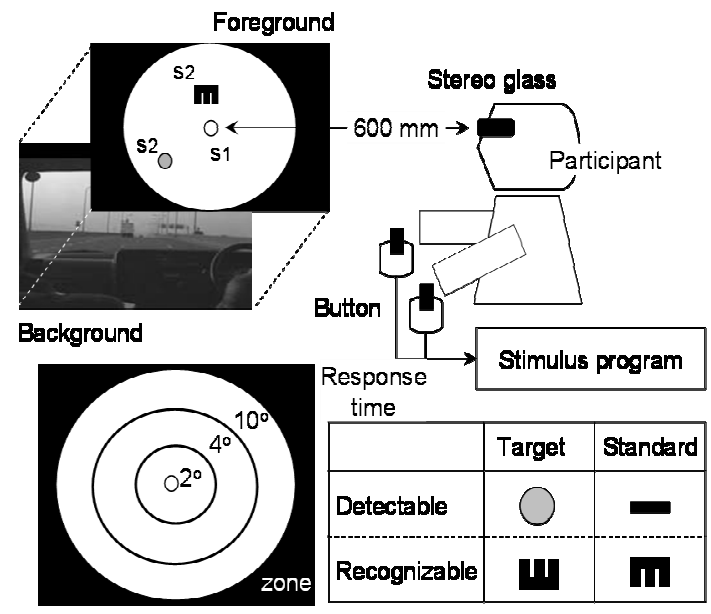

Figure 6. Experimental procedure.

3D driving scenes were used for distracting the visual attention and interfering with recognition. Four patterns of driving scenes were used, as shown in Fig. 7, where V and D denote the factors of velocity and demand of traffic. There were two levels for factor V (velocity of vehicle: $\mathrm{V} 1=40$ $\mathrm{km} / \mathrm{h}, \mathrm{V} 2=80 \mathrm{~km} / \mathrm{h}$ ) and factor $\mathrm{D}$ (demand of traffic: $\mathrm{D} 1=$ non-crowded, D2=crowded). 


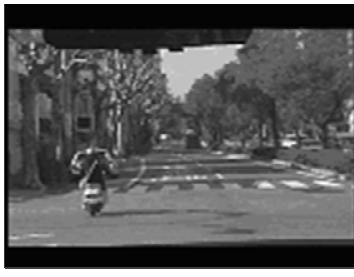

V1 and D1

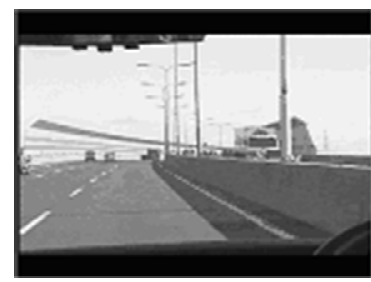

V2 and D1

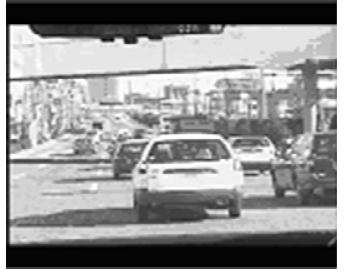

V1 and D2

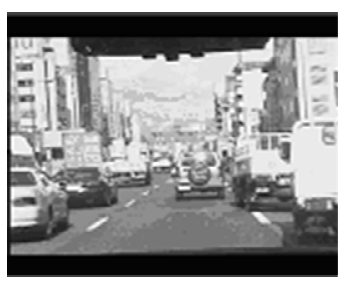

V2 and D2
Figure 7. Driving scene as background.

Two experiments were carried out in order to examine the effects of these factors. In one experiment, we measured the detection region of the simple white circle S1 ("detection experiment"), and in the other ("recognition experiment") we measured the recognition region with respect to a specific shape in the Snellen chart (S2). In the experiments, the participants were 17 university students aged 20 to 23 who had regular driver's licenses and had normal or corrected-to-normal vision.

S2 appeared 48 times per run in the detection experiment and 256 times per run in the recognition experiment. Furthermore, the standard mark appeared 208 times, and the target indicator appeared 48 times. In the experiments, the mark S2 appeared the same number of times per zone, and the difference in luminosity of S2 to the background was determined as 101x.

The response time is shown in Fig. 8 for the region where the target mark S2 appeared, where it is clear there were no differences between the regions. On the other hand, in the recognition experiment, there is a clear tendency that a wider regions of appearance of target mark S2 entailed a longer response time. For three regions of appearance, the response time for recognition was longer than that for detection, with a difference ranging between 230 and $290 \mathrm{~ms}$ on average.

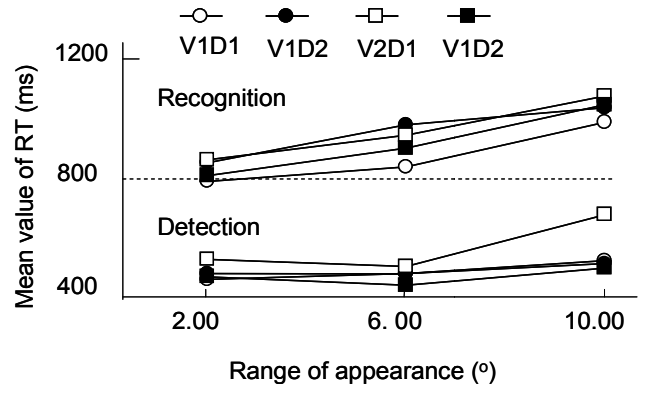

Figure 8. RT and range of appearance.

\subsection{Useful Field of View [16,17]}

The UFOV is the region where a participant can distinguish the shape, direction, and other parameters of the mark. This region is located in the central area, as compared with the static/kinetic field or conspicuity field, as shown in Fig. 9. In this figure, the field of view is defined by the region around the fixation point (FP) [10]. A problem is that there are no standards on how to measure UFOV at the fovea with a visual task. The term UFOV generally implies that peripheral performance depends not only on retinal sensitivity, but also on the nature of the perceptual task. However, since these fields vary greatly with respect to differences in shape between target and background, density of background noise, and so forth, it is extremely difficult to measure the boundaries exactly. In a recent study, Ball [12] measured the region as the UFOV where the target index can be detected against a unique or ambiguous background. Also, Ikeda [11] evaluated the influence of fovea load on UFOV. However, a quantitative guarantee cannot be given for the degree of accuracy of the UFOV as obtained above.

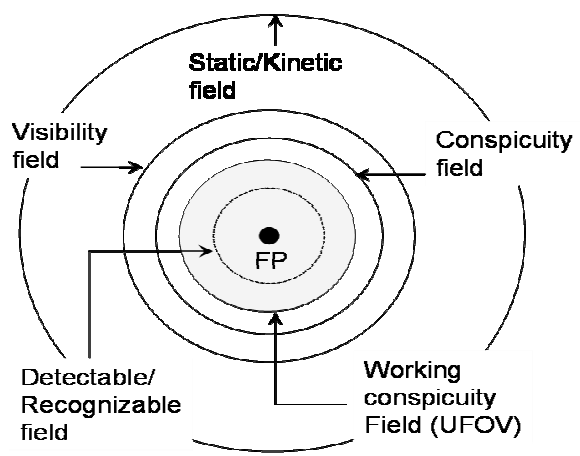

Figure 9. Definitions of field of view [10].

In the following, we demonstrate a new method of measuring UFOV developed by the authors and the results of its application, where the same experimental devices as in the experiment on RT in Fig. 5 are used. The experimental paradigm is shown in Fig. 10. A simple black circle (fixation point $\mathrm{S} 1$ ) with a visual angle of $0.86^{\circ}$ was used to keep the visual attention at the center of the CRT display. The recognition mark (S2), also with a size of $0.86^{\circ}$ appeared in eight different orientations $\left(0^{\circ}, 45^{\circ}, 90^{\circ}, 135^{\circ}, 180^{\circ}, 225^{\circ}\right.$, $\left.270^{\circ}, 315^{\circ}\right) . \mathrm{S} 2$ is a specific object such as a character, an arrow or a certain shape, which are three standard types of indicators with the same attributes as shown in Fig. 11. The reason why these three types of recognition indicators are chosen is to investigate the differences in human performance with regard to different visual stimuli.

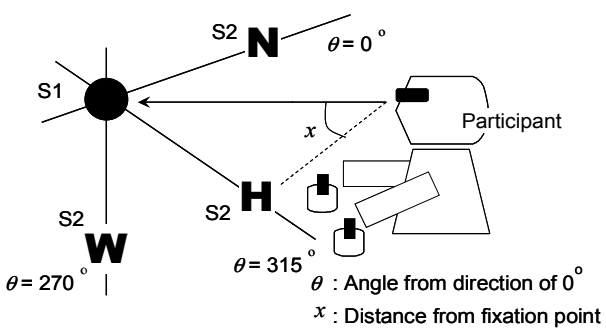

Figure 10. Distance and angles of recognition index. 


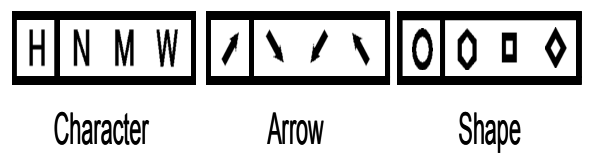

Figure 11. Recognition indices.

In the experiments, we measured two types of response categories, namely "possible to detect" or "impossible to detect" for the distance from the fixation point S1 to the recognition mark S2. As shown in Fig. 12, the distance from the fixation point to the recognition mark indicates the strength of the stimulus as a psychophysical quantity. Whereas the recognition mark can be detected when it appears near the fixation point, and it becomes increasingly difficult and eventually impossible to detect the recognition mark when it appears further away from the fixation point. In other words, a longer distance from the fixation point to the recognition mark corresponds to a weaker strength of the stimulus.
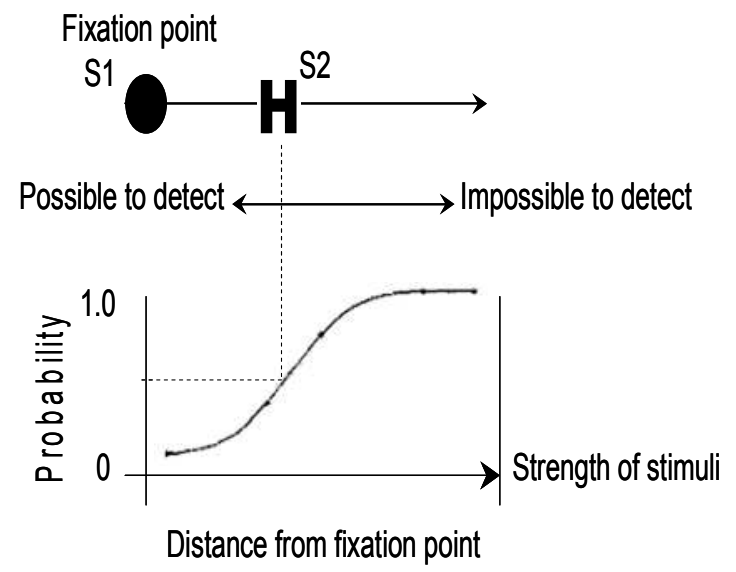

Figure 12. Psychometric curve.

The purpose of this experiment is to measure the threshold of recognition when the response category changes from "possible to detect" to "impossible to detect" or vice versa. It is well known that the function linking the possibility of detection and the strength of the stimulus can be obtained as a psychometric curve [10]. Since the distance of the boundary between the "possible to detect" and "impossible to detect" categories indicates the threshold of recognition, the latter can be estimated by using a psychometric curve. Therefore, it may be possible to obtain the psychometric curves in any direction from the fixation point, and it is also known that the stimulus threshold can be obtained as a probabilistic percentile of this psychometric curve. One of the examples of this threshold with a $50 \%$ probability is the point of subjective equality (PSE), which is equivalent to the threshold of recognition. As shown in Fig. 13, if we can assume that the region plotted within these stimulus thresholds is defined as UFOV, then the outer limit of the region connected with PSE for each angle is also defined as UFOV.

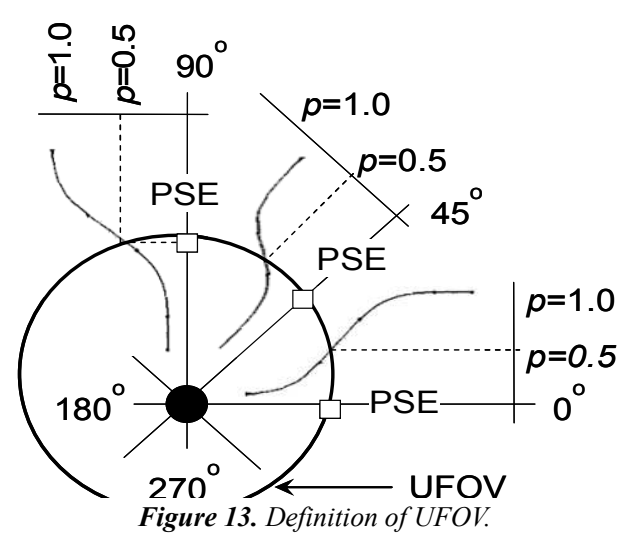

Fig. 14(a) shows the kinetic field of view of participant B. In this figure, the regions shown in solid and dotted lines are the fields for the recognition marks with a size of $0.86^{\circ}$ and $0.43^{\circ}$ respectively. On the other hand, the regions shown in Fig. 14(b) are the UFOV for the recognition marks with a size of $0.86^{\circ}$ and $0.43^{\circ}$, respectively. Compared with Fig. 14 (a) and (b), it is clear that there is a difference in the region between the kinetic field of view and UFOV. From Fig. 14 (b), it can be seen that UFOV is affected by the size of the recognition mark, and this tendency is observed for all types of recognition marks and all participants. These facts hint at important differences in the mechanism of IPS for the kinetic field of view and UFOV. In the former case, there are no differences caused by the size of the target mark since the mechanism of IPS is simple, such as in the skill base under the operator model of Fig. 2. This means that there is little variation in kinetic field in the visual task. On the other hand, the variation in UFOV means that the mechanism in this case is due to a knowledge-based task in the operator model. Therefore, the deterioration in human performance in the case of the kinetic field is small, and therefore the probability of failure due to human error is small in comparison with that in the case of UFOV. A plot of RT as a function of UFOV is also shown in Fig. 15, which clearly shows that there is correlation between the two parameters $(r=0.79)$, although the correlation between RT and UFOV is a useful tool for estimating the probability of failure caused by human error.

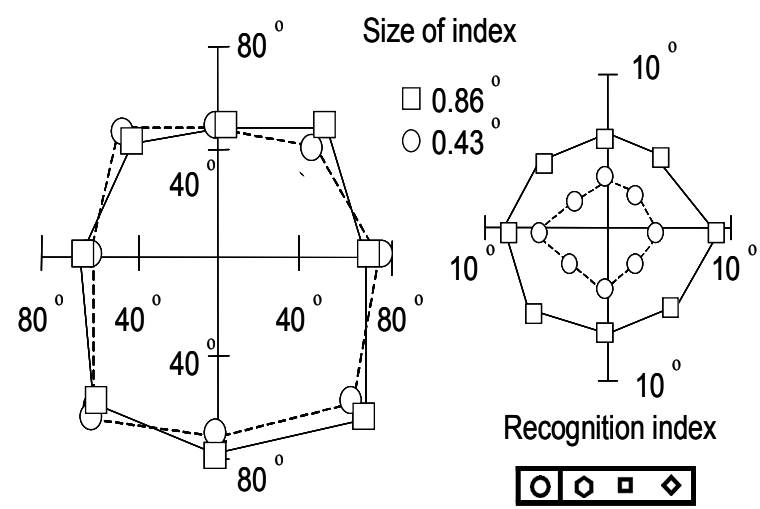

(a) Kinetic field

(b) Useful field of view

Figure 14. Comparison of UFOV with Kinetic field (participant B 


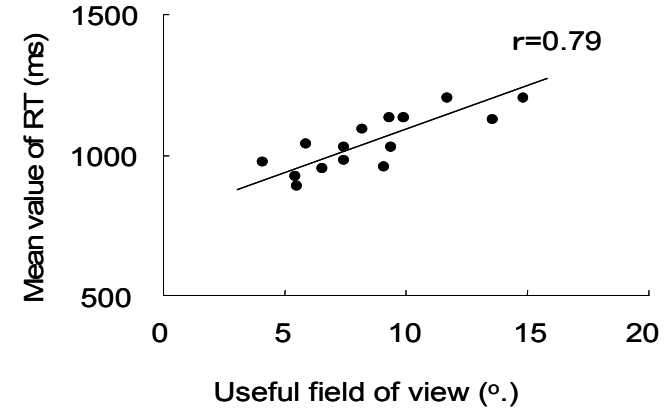

Figure 15. Relation between RT and UFOV.

\section{Discussion}

In this section, we assume that there are several relationships between human perceptual performance and safety. Therefore, this question is discussed by considering the broad fields of psychology and cognitive science.

\subsection{Experimental Results and Hypotheses}

The phenomenon of narrowing of the region within the visibility field as a result of additional visual tasks (known as visual tunneling, perceptual narrowing or tunnel vision), is highly relevant to information processing systems (IPS). In order to explain the mechanism of this phenomenon, a new hypothesis on visual attention has been proposed from the viewpoint of cognitive and psychophysical science. In this hypothesis, the reasons for the occurrence of visual tunneling are based on the following two distinct rules $[4,6,8]$ :

Rule 1: When the visual demand exceeds the ordinal visual task, the distance of moving visual attention is out of the region of the useful field of view.

Rule 2: When the visual demand exceeds the ordinal visual task, there is less overlap with UFOV.

Fig. 16 shows an illustration of the concept of these rules, which is derived from the concept of the depth $\mathrm{L}$ and width $\mathrm{W}$ of visual processing and the principle of constant resources for information processing. Specifically, this hypothesis can be easily applied to general situations with moving fixation points by changing the moving distance D in Fig. 16.

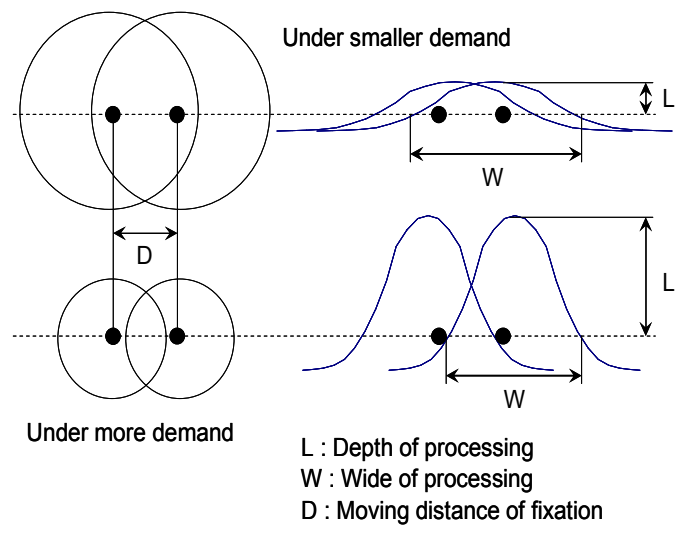

Figure 16. Visual tunneling by psychophysical model [6].
In addition, a new concept referred to as cognitive momentum, which indicates the active characteristics for information processing, was recently proposed[8,9]. According to cognitive momentum, the reason why visual tunneling occurs at higher visual demand is not the degradation of information processing but rather the adaptation to the changed situation for information processing caused by partitioning of resources for information processing.

\subsection{Cognitive Model with Processing Mechanism}

The typical results of the experiments mentioned in the previous section raise two important questions, namely, (1) why there is a difference of 230 to $290 \mathrm{~ms}$ in response time and a difference of $1.5^{\circ}$ to $3.15^{\circ}$ in angle between detection and recognition experiments, as shown in this experimental paradigm, and (2) how we can explain the existence of several types of distinct response times depending on the zone $(1,2$ or 3$)$ in the recognition experiment.

The most pervasive model of a human operator is likely the S-O-R paradigm [2], where the response is assumed to be a function of the stimulus and the organism, that is,

$$
R=f(S, O)
$$

The Rasmussen model [1] is one such model which became well-known as the SLM in cognitive science [1]. It is nevertheless difficult to appreciate that the S-O-R paradigm and SLM show two fundamental similarities, such as sequential progression through the internal mechanism and dependence on a stimulus or event to start the processing. Therefore, it is difficult to answer the above two questions by using cognitive models such as the S-O-R paradigm or SLM. One of the latest versions of this metaphor is based on the notion of human cognition as a fallible machine. This idea is most clearly expressed through an indirect definition of a feasible information processing device [1].

It is well known from brain research that there are two channels of visual information processing, where one channel concerns the detection of motion and the other concerns the recognition of the characteristics of objects [7]. From this knowledge, we can construct a model of the mechanism of information processing corresponding to the experimental paradigm in this paper. Fig. 17 shows an IPS model for a visual task proposed by the authors [13], where the two channels correspond to information processing of foreground and background scenes respectively. As shown in the figure, the processing in both experiments (detection and recognition) is performed in parallel. Therefore, more time is required for recognition in the recognition experiment when presented with a visual task involving a target object located far from the fixation point, which answers both questions. 


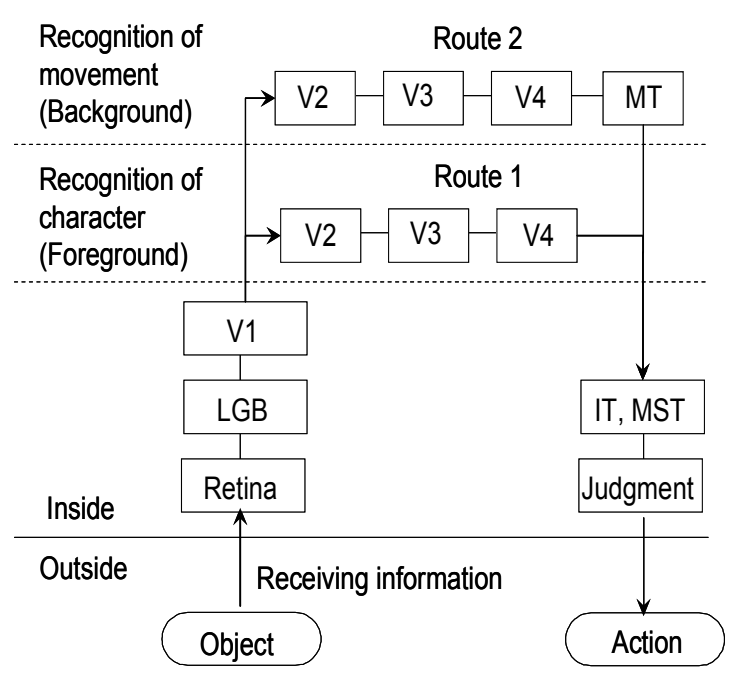

Figure 17. IPS model for visual task.

\subsection{Effect Of UFOV and RT on Structural Safety}

The performance with respect to two typical types of human perception was investigated. As a result, deterioration in performance was confirmed, and the reasons were explained by the IPS model which takes into account the psychological, cognitive, and physiological aspects of the mechanism of perception. To predict the effect of deterioration in perceptual ability on structural safety, we consider the causal relation between changes in UFOV and response time as shown in Fig. 18. A wider UFOV means that the moving eyes are not fixated since the peripheral field of view is wider when UFOV is wide in the case of moving eyes. However, we can assume that the visual task requires a longer time to complete with a wide UFOV than with a narrow UFOV since there are more resources for IPS in the former case at the same depth $\mathrm{L}$ for information processing in Fig. 16.

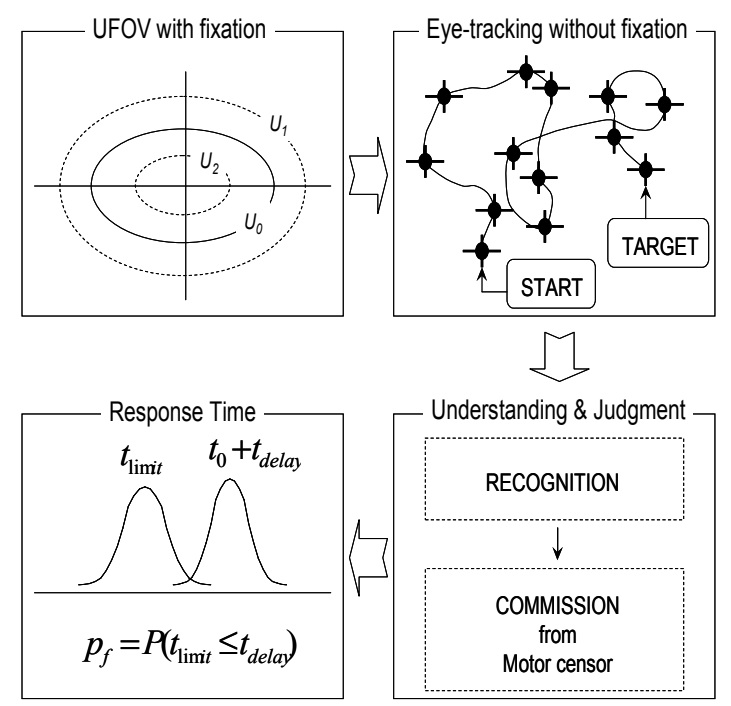

Figure 18. Structural safety and human perceptual properties.
As shown in Fig. 18, it may be possible to estimate the probability of failure by calculating the relation between the response time and the delay in response time. However, as shown in Fig. 15, it is difficult to obtain an exact correspondence in this relationship. This problem will be investigated in future work.

As mentioned in the discussion in Section 2, the strength of human-machine systems is determined by the strength of the machine and the human performance. However, there are substantial differences between the characteristics of subjective uncertainties of machine strength and human performance. In the former, the strength $\mathrm{Rm}$ of the machine is independent of the applied stress, whereas the human performance $\mathrm{Rh}$ is.

The difficulties of estimating the degree of degradation in strength of human-machine systems are due to these dependences and nonlinear behavior. This relation is illustrated in Fig. 19, where the strength distribution of human-machine systems is independent of the subjective uncertainty associated with machines and dependent on that associated with human performance. Based on the discussion in this section, these relations affect the final probability of failure of structural systems, including human-machine systems, as illustrated in Fig. 20.

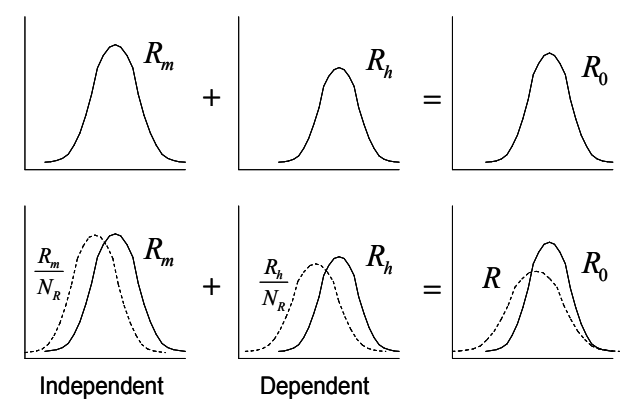

Figure 19. Dependency and independency of strength distribution.

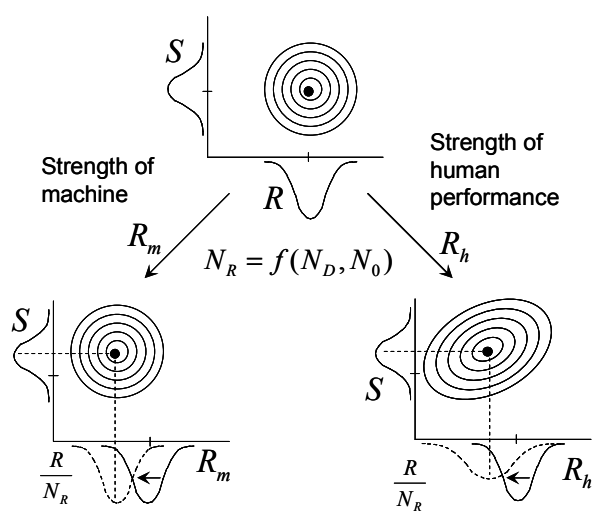

Figure 20. Effect of subjective uncertainty of machine and human performance.

\section{Conclusion}

The relation between structural safety and the performance of the operator in terms of visual perception was investigated by focusing on the parameters of response time (RT) and the useful field of view (UFOV) in order to eva- 
luate the effects of deterioration of human performance in visual tasks. It is also considered that there are several relationships between human perceptual performance and structural safety in human-machine systems. The causes for deterioration of human performance, such as the properties of human perception, are discussed on the basis of an IPS model. These issues will be the focus of future work, whereby it is necessary to formulate a method for measuring and standardizing human performance. Furthermore, to evaluate the effect of changes in human performance, the method for estimating structural reliability and safety must be linked with the method for estimating human performance.

\section{References}

[1] J. Reason, Human Error, Cambridge, UK: Cambridge University Press, 1990.

[2] E. Hollnagel, Human Reliability Analysis -Context and Control, Massachusetts, USA: Academic Press, 1993.

[3] S.K. Card, T.P. Noran, and A. Newell, The Psychology of Human-Computer Iteraction, New Jersey, USA: Lawrence Erlbaum Associates Publishers, 1983.

[4] T. Miura, "What is the narrowing of visual field with the license of increase of speed", Proc. of 10th International Congress on Association for Accident and Traffic Medicine, 1985, pp.21-24.

[5] K. Samnel, L. Yan and P. Marianna, The Stress-Strength Model and Its Generalizations: Theory and Application, Toh Tuck Link, Singapore: World Scientific Publishers, 2003.

[6] T. Miura, "Visual search in Intersections -An Underlying Mechanism-“, IATSS Research, Vol.16, No.1, 1992, pp.42-49.

[7] S. Amari, and K. Toyama, Dictionary of Brain Science, Tokyo, Japan: Asakura Publiser Inc., 2000.

[8] T. Miura, Behavior and Visual Attention, Tokyo, Japan:
Kazama Publisher Inc., 1996.

[9] T Miura, K Shinohara and K Kanda, "Visual Attention in Automobile driving: From Eye Movement Study to Depth Attention Study", Proc. of 2nd International Conference on Psychophysiology in Ergonomics, 1988, pp.7-8.

[10] T. Ohyama, S. Imai and T.Wake, Handbook of Sensory and Perceptive Psychology, Tokyo, Japan: Seishinn Publisher Inc., 1994.

[11] M. Ikeda and T. Takeuchi, "Influence of foveal load on the functional visual field". Perception \& psychophysics, Vol.18, No.4, 1975, pp.225-260.

[12] K. Ball and C.Owsley, "The useful field of view test: A new technique for evaluating age related declines in visual function". Journal of American Optometric Association, Vol.16, No.1, 1993, pp.71-79.

[13] K. Yamanaka and H. Nakayasu, "A model of information processing for visual task at driving a load vehicle", Proc. of 3rd International Conference Artificial Intelligence and Applications, 2003, pp.545-550.

[14] K. Yamanaka, H. Nakayasu and K. Maeda, "Experimental Study on Visual Information Transition at Driving Automobile for Reliability-Based Design", Proc. of Workshop on Reliability-Based Design and Optimization, 2002, pp.251-256.

[15] K. Yamanaka, K. Maeda, T. Miyoshi, H. Hayashi and H. Nakayasu, "Evaluation of response properties an automobile driver based on design of experiment", Advances in Transportation Studies an International Journal, Section B6, 2005, pp.37-55.

[16] K. Yamanaka, H. Nakayasu and T. Miyoshi, "Recognition-Probability-Based Useful Field of View by Probit Method", Proc. of 13th International Workshop on Robot and Human Interactive Communication, 2004, pp.607-612.

[17] K. Yamanaka and H. Nakayasu, "Measurement and Evaluation of Functional Visual Field in Practical Task". Proc. of 2nd International Symposium on Measurement Analysis and Modeling of Human Functions, 2004, pp.407-412. 